Acta Crystallographica Section E

Structure Reports

Online

ISSN 1600-5368

\section{3-(2-Formylphenoxy)propanoic acid}

\section{Alain Collas, Christophe M. L. Vande Veldeł and Frank Blockhuys*}

Department of Chemistry, University of Antwerp, Universiteitsplein 1, B-2610 Wilrijk, Belgium

Correspondence e-mail: frank.blockhuys@ua.ac.be

Received 13 September 2010; accepted 23 September 2010

Key indicators: single-crystal X-ray study; $T=293 \mathrm{~K}$; mean $\sigma(\mathrm{C}-\mathrm{C})=0.004 \AA$; $R$ factor $=0.047 ; w R$ factor $=0.119 ;$ data-to-parameter ratio $=10.3$.

In the structure of the title compound, $\mathrm{C}_{10} \mathrm{H}_{10} \mathrm{O}_{4}$, the carboxyl group forms a catemer motif in the [100] direction instead of the expected dimeric structures. The carboxylic acid group is found in the syn conformation and the three-dimensional organization in the crystal is based on $\mathrm{C}-\mathrm{H} \cdots \mathrm{O}$ and $\mathrm{O}-$ $\mathrm{H}$... $\mathrm{O}$ interactions.

\section{Related literature}

For the synthesis, see: Zawadowska (1963); Jarvest et al. (2005). For related structures, see: Gresham et al. (1949); Leiserowitz (1976); Borthwick (1980); Kennard et al. (1982); Shockravi et al. (2004); Gao \& Ng (2006). For applications of poly( $p$-phenylene vinylene) oligomers (PPVs), see: Chemla (1987); Bandyopadhyay \& Pal (2003). For hydrogen bonding and crystal engineering, see: Desiraju (1997); Steiner (2002).

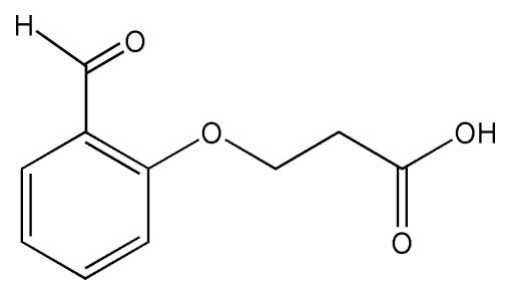

\section{Experimental}

\section{Crystal data}

$\mathrm{C}_{10} \mathrm{H}_{10} \mathrm{O}_{4}$

$M_{r}=194.18$

Orthorhombic, $\mathrm{Pbca}$

$a=15.269$ (4) $\AA$

$b=7.167$ (2) $\AA$

$c=17.136(5) \AA$

Data collection
Enraf-Nonius CAD-4

diffractometer

1718 measured reflections

1718 independent reflections

Refinement

$R\left[F^{2}>2 \sigma\left(F^{2}\right)\right]=0.047$

$w R\left(F^{2}\right)=0.119$

$S=1.02$

1718 reflections
964 reflections with $I>2 \sigma(I)$

3 standard reflections every $60 \mathrm{~min}$ intensity decay: $1 \%$
Table 1

Hydrogen-bond geometry $\left(\AA{ }^{\circ}\right)$.

\begin{tabular}{lllll}
\hline$D-\mathrm{H} \cdots A$ & $D-\mathrm{H}$ & $\mathrm{H} \cdots A$ & $D \cdots A$ & $D-\mathrm{H} \cdots A$ \\
\hline $\mathrm{O} 3-\mathrm{H} 41 \cdots \mathrm{O} 4^{\mathrm{i}}$ & $0.94(4)$ & $1.72(4)$ & $2.618(3)$ & $158(3)$ \\
$\mathrm{C} 21-\mathrm{H} 21 a \cdots{ }^{\mathrm{ii}}$ & $1.00(3)$ & $2.64(3)$ & $3.409(4)$ & $134.5(2)$ \\
$\mathrm{C} 22-\mathrm{H} 22 b \cdots \mathrm{O} 1^{\text {iii }}$ & $0.96(3)$ & $2.46(3)$ & $3.187(4)$ & $132(2)$ \\
$\mathrm{C} 21-\mathrm{H} 21 a \cdots 3^{\text {iv }}$ & $1.00(3)$ & $2.60(2)$ & $3.456(3)$ & $144(2)$ \\
$\mathrm{C} 3-\mathrm{H} 3 \cdots 4^{\mathrm{v}}$ & $0.96(2)$ & $2.71(2)$ & $3.536(4)$ & $144.9(2)$ \\
\hline
\end{tabular}

Symmetry codes: (i) $-x-\frac{1}{2}, y-\frac{1}{2}, z$; (ii) $-x,-y+1,-z+1$; (iii) $x,-y+\frac{1}{2}, z-\frac{1}{2}$; (iv) $-x, y+\frac{1}{2},-z+\frac{1}{2} ;(\mathrm{v}) x+\frac{1}{2}, y,-z+\frac{1}{2}$.

Data collection: CAD-4 EXPRESS (Enraf-Nonius, 1994); cell refinement: CAD-4 EXPRESS; data reduction: XCAD4 (Harms \& Wocadlo, 1995); $\operatorname{program}(\mathrm{s})$ used to solve structure: SHELXS97 (Sheldrick, 2008); program(s) used to refine structure: SHELXL97 (Sheldrick, 2008); molecular graphics: ORTEP-3 (Farrugia, 1997) and PLATON (Spek, 2009); software used to prepare material for publication: Win $G X$ (Farrugia, 1999).

CVV thanks the Fund for Scientific Research (FWO Vlaanderen) for a grant as a research assistant. AC wishes to thank the Institute for the Promotion of Innovation by Science and Technology in Flanders (IWT) for a predoctoral grant.

Supplementary data and figures for this paper are available from the IUCr electronic archives (Reference: ZL2308).

\section{References}

Bandyopadhyay, A. \& Pal, A. J. (2003). Appl. Phys. Lett. 82, 1215-1217.

Borthwick, P. W. (1980). Acta Cryst. B36, 628-632.

Chemla, D. S. (1987). Nonlinear Optical Properties of Organic Molecules and Crystals. Boston: Academic Press.

Desiraju, G. R. (1997). Chem. Commun. 16, 1475-1482.

Enraf-Nonius (1994). CAD-4 EXPRESS. Enraf-Nonius, Delft, The Netherlands.

Farrugia, L. J. (1997). J. Appl. Cryst. 30, 565.

Farrugia, L. J. (1999). J. Appl. Cryst. 32, 837-838.

Gao, S. \& Ng, S. W. (2006). Acta Cryst. E62, o3420-03421.

Gresham, T. L., Jansen, J. E., Shaver, F. W., Bankert, R. A., Beears, W. L. \& Pendergast, M. G. (1949). J. Am. Chem. Soc. 71, 661-663.

Harms, K. \& Wocadlo, S. (1995). XCAD4. University of Marburg, Germany. Jarvest, R. L., Erskine, S. G., Forrest, A. K., Fosberry, A. P., Hibbs, M. J., Jones, J. J., O'Hanlon, P. J., Sheppard, R. J. \& Worby, A. (2005). Bioorg. Med. Chem. Lett. 15, 2305-2309.

Kennard, C. H. L., Smith, G. \& White, A. H. (1982). Acta Cryst. B38, 868-875. Leiserowitz, L. (1976). Acta Cryst. B32, 775-802.

Sheldrick, G. M. (2008). Acta Cryst. A64, 112-122.

Shockravi, A., Alizadeh, R., Aghabozorg, H., Mohebbi, L., Moradi, K. S. \& Moghimi, A. (2004). Iran. J. Chem. Chem. Eng. 23, 37-44.

Spek, A. L. (2009). Acta Cryst. D65, 148-155.

Steiner, T. (2002). Angew. Chem. Int. Ed. 41, 48-76.

Zawadowska, I. (1963). Acta Pol. Pharm. 20, 25-30.

¥ Current address: Karel de Grote University College, Department of Applied Engineering, Salesianenlaan 30, B-2660 Antwerp, Belgium. 


\section{supporting information}

Acta Cryst. (2010). E66, o2662 [doi:10.1107/S1600536810038079]

\section{3-(2-Formylphenoxy)propanoic acid}

\section{Alain Collas, Christophe M. L. Vande Velde and Frank Blockhuys}

\section{S1. Comment}

The title compound was synthesized as a precursor for asymmetric PPV-type [poly(p-phenylene vinylene)] oligomers. These compounds are promising candidates as the active materials in organic memories (Bandyopadhyay \& Pal, 2003) and as non-linear optical (NLO) materials with a high first-order hyperpolarizability (Chemla, 1987). Besides the condition that these oligomers should bear donor and acceptor substituents connected by a $\pi$-system, it is of critical importance for their usefulness as an NLO material and as a bistable organic memory material that the crystal packing is non-centrosymmetric. In particular, the compound should crystallize in a polar space group. To influence the crystal packing by means of the crystal engineering methodology in order to meet this criterion, it is necessary to introduce certain statistically well chosen synthons (Desiraju, 1997). Therefore, we opted for carboxylic acid functional groups (powerful hydrogen bond donors) in the basic structure of the organic semiconductor. To combine the electronic (A- $\pi$-D) and the structural (non-centrosymmetric space group) requirements, we used the Williamson ether synthesis to prepare the title compound as a building block for a PPV-based semiconductor bearing a carboxylic acid moiety.

The geometry of the aldehyde contains no surprises. The methylene fragments are in synclinal conformation and the torsion angle is $65.9(3)^{\circ}$. As a result and due to the repulsion between the lone pairs of oxygen atoms $\mathrm{O} 2$ and $\mathrm{O} 3$, the carboxylic acid group, found in the syn conformation, is twisted out of the plane of the phenyl ring. The $\mathrm{O} 2 \cdots \mathrm{O} 3$ distance is 3.034 (3) $\AA$ and this is the only contact of the ether oxygen atom. The distances in the carboxylic acid moiety are in accordance with the expected values for non-disordered carboxylic acids (Leiserowitz, 1976): the carbonyl $(\mathrm{C}=\mathrm{O})$ bond length $\mathrm{C} 23-\mathrm{O} 4$ is 1.209 (3) $\AA$ and the $\mathrm{C}-\mathrm{O}$ single bond length $\mathrm{C} 23-\mathrm{O} 3$ is 1.306 (3) $\AA$. The angles of the carboxyl moiety contain no surprises (Borthwick, 1980): $\mathrm{C} 23-\mathrm{O} 3-\mathrm{H} 31$ is $110(2)^{\circ}$ and $\mathrm{O} 4-\mathrm{C} 23-\mathrm{O} 3$ is $121.9(2)^{\circ}$.

Two intramolecular $\mathrm{CH} \cdots \mathrm{O}$ hydrogen bonds involving the aldehyde group can be identified: the relevant parameters of $\mathrm{C} 6-\mathrm{H} 6 \cdots \mathrm{O} 1$ and $\mathrm{C} 11-\mathrm{H} 1 \cdots \mathrm{O} 2$ are given in Table 1, entries 1 and 2 . The incorporation of a carboxylic acid group was expected to yield the corresponding dimer synthon, but the crystal structure of the title compound reveals a catemer motif

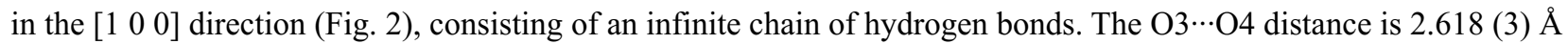
(Table 1, entry 3 ) which is not surprising for a hydrogen bond of moderate strength (Steiner, 2002). The chains are intertwined through $\mathrm{O} 1 \cdots \mathrm{Cg}$ contacts in which the aldehyde oxygen atom contacts the center of the aromatic ring $\left[\mathrm{O} 1 \cdots C g^{\mathrm{i}} 3.508(3) \AA, \mathrm{C} 11-\mathrm{O} 1 \cdots C g^{\mathrm{i}} 78.47(17)^{\circ}\right.$, symm. code $\left.\mathrm{i}=1-x,-y,-z\right]$. $\mathrm{O} 1$ is also involved in weak $\mathrm{CH} \cdots \mathrm{O}$ hydrogen bonds with $\mathrm{H} 21 a$ (Table 1, entry 4 ) and $\mathrm{H} 22 b$ (Table 1, entry 5); the latter links the infinite hydrogen bonded

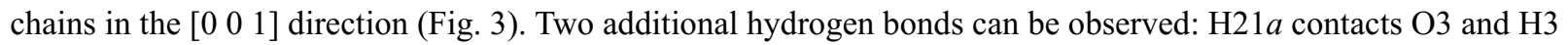
contacts $\mathrm{O} 4$ (Table 1, entries 6 and 7, respectively).

\section{S2. Experimental}

Salicylic aldehyde and 3-chloropropanoic acid were obtained from ACROS and used as received. The general procedure of Gresham was followed (Gresham et al., 1949). $8 \mathrm{~g}$ of $\mathrm{NaOH}$ in $20 \mathrm{ml}$ of distilled water were added to a stirred solution 
of $12.2 \mathrm{~g}(0.1 \mathrm{~mol})$ of salicylic aldehyde and $10.9 \mathrm{~g}$ ( $0.1 \mathrm{~mol})$ of 3-chloropropanoic acid in $80 \mathrm{ml}$ of distilled water. After heating under reflux for $4 \mathrm{~h}$, the mixture was acidified with $19.5 \mathrm{ml}$ of conc. $\mathrm{HCl}$. Unreacted salicylic aldehyde was removed by steam distillation and the resulting mixture was placed in the refrigerator. The resulting tan-coloured needles were collected by filtration in a $20 \%$ yield. M.p. $381 \mathrm{~K}$ (uncorrected). Crystals suitable for the diffraction experiment were grown by slow evaporation of a 50:50 chloroform:toluene solvent mixture, analogous with the experiments of Kennard (Kennard et al., 1981). ${ }^{1} \mathrm{H}$ NMR ( $\left.\mathrm{CD}_{3} \mathrm{OD}, 400 \mathrm{MHz}, \mathrm{TMS}\right): \delta 2.84$ (t, $\left.2 \mathrm{H}, 6.00 \mathrm{~Hz}, \mathrm{H} 22\right), 4.39$ (t, $2 \mathrm{H}, 6.00 \mathrm{~Hz}$, H21), 7.05 (td, 1H, 7.44 and $0.88 \mathrm{~Hz}, \mathrm{H}$ ), 7.18 (d, 1H, $8.10 \mathrm{~Hz}, \mathrm{H} 3), 7.61$ (td, 1H, 7.32 and $1.88 \mathrm{~Hz}, \mathrm{H} 4), 7.75$ (dd, 1H, 7.72 and $1.80 \mathrm{~Hz}, \mathrm{H} 6), 10.39(\mathrm{~d}, 1 \mathrm{H}, 0.80 \mathrm{~Hz}, \mathrm{H} 21 \mathrm{~b})$; the proton of the carboxylic acid can not be seen. ${ }^{13} \mathrm{C}$ NMR ( $\left.\mathrm{CDCl}_{3}, 100 \mathrm{MHz}, \mathrm{TMS}\right): \delta 34.17$ (C22), 63.83 (C21), 112.66 (C3), 121.33 (C5), 125.08 (C1), 128.61 (C6), 135.99 (C4), 160.75 (C2), 175.97 (C11), 189.79 (C23).

\section{S3. Refinement}

The positions of the hydrogen atoms were derived from the electron density difference map and $X-\mathrm{H}$ bonding distances are situated between 0.89 (3) $\AA$ and 1.03 (3) $\AA$.

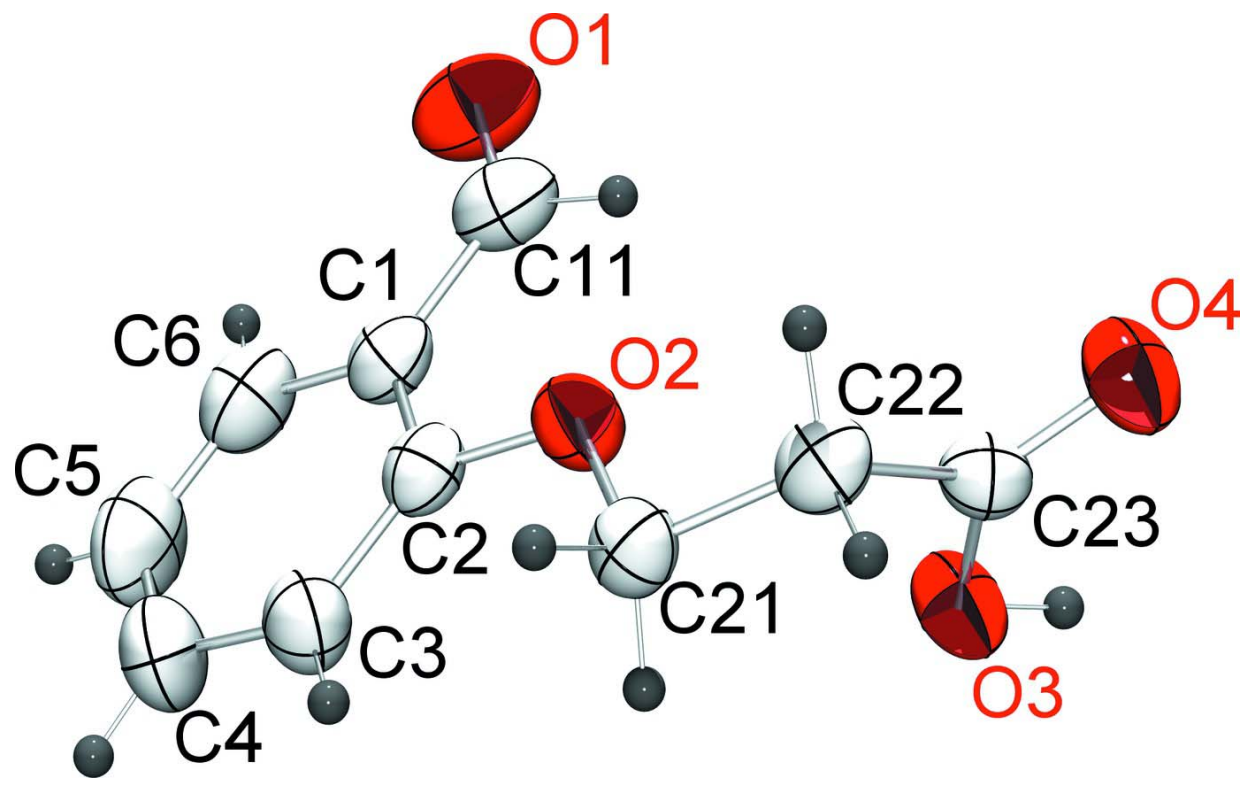

\section{Figure 1}

Molecular structure of the title compound with the numbering scheme. Displacement ellipsoids are drawn at the 50\% probability level and hydrogen atoms are represented as spheres with an arbitrary radius. 


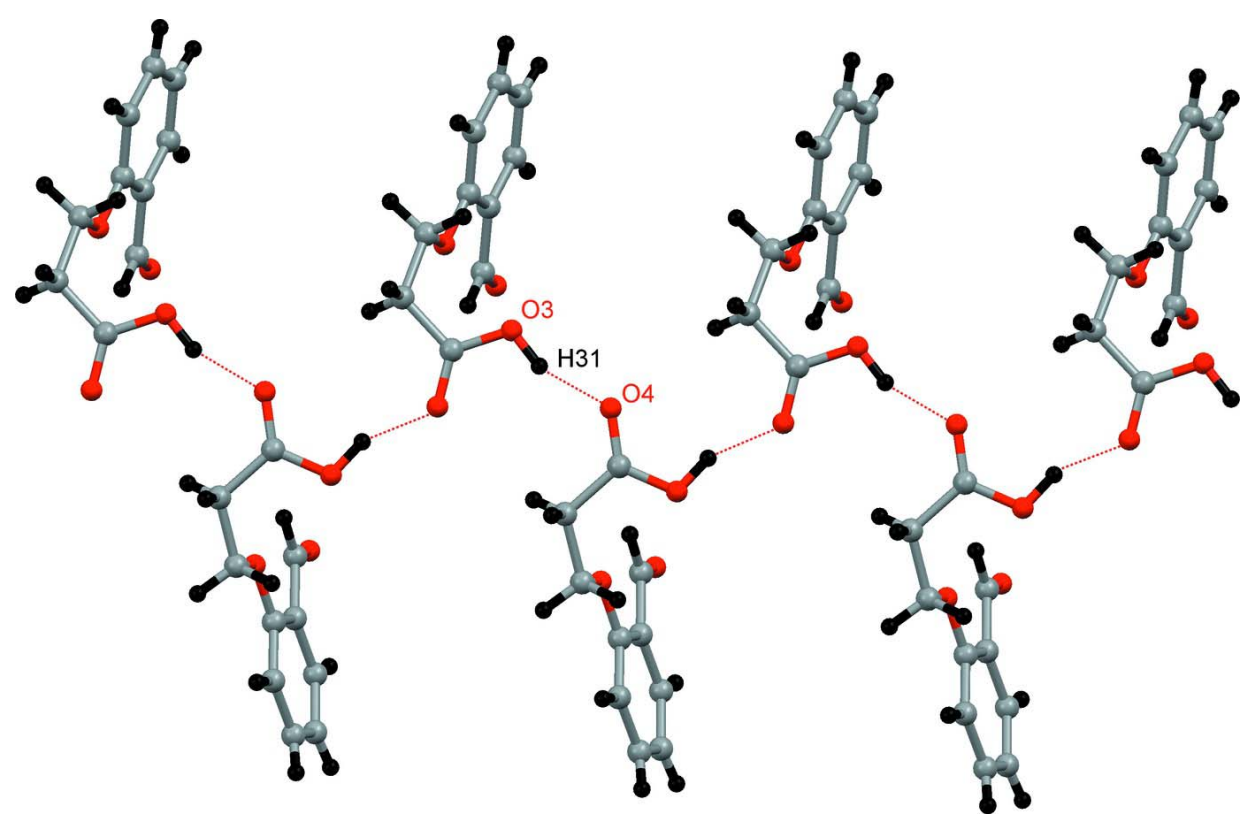

\section{Figure 2}

Representation of the infinite chain of hydrogen bonds involving the carboxyl moiety through the crystal structure of the title compound. See Table 1 for details.

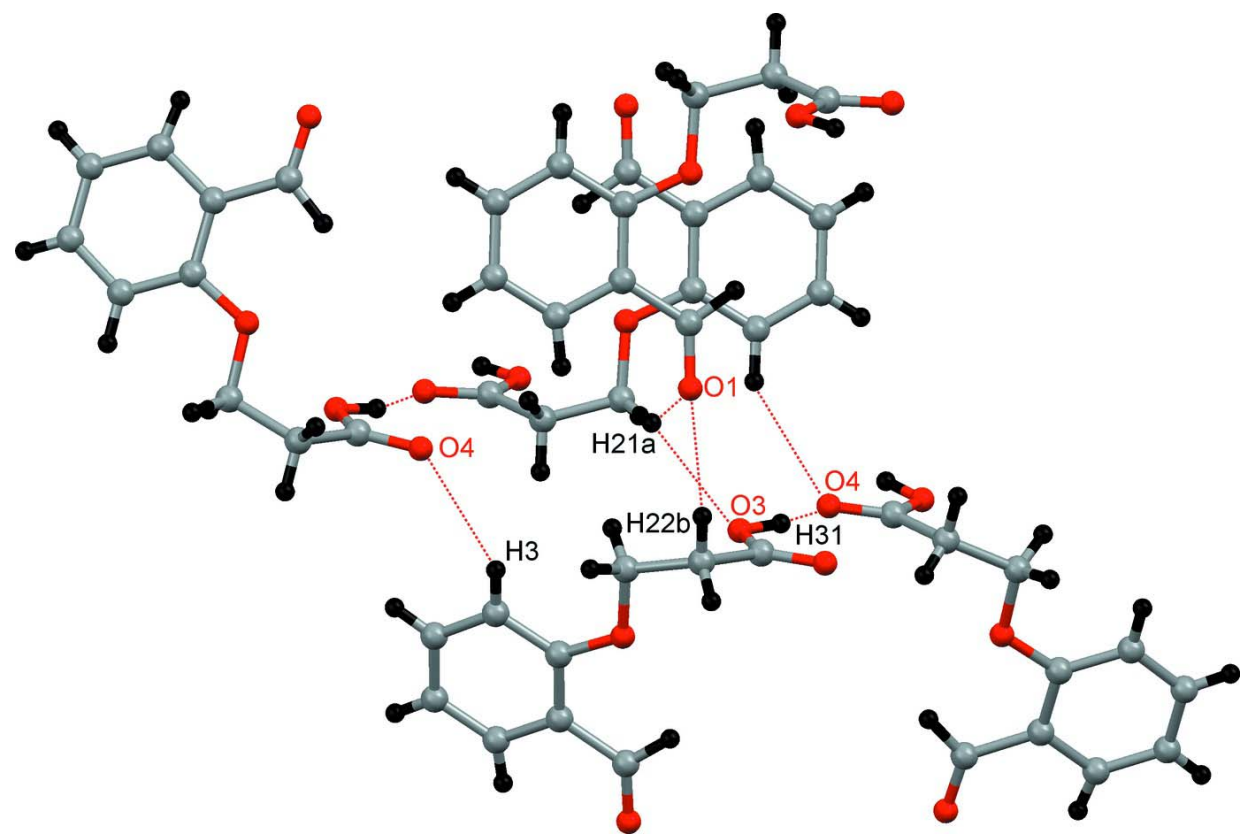

\section{Figure 3}

Additional short contacts in the crystal structure of the title compound. See Table 1 for details.

\section{3-(2-formylphenoxy)propanoic acid}

Crystal data

$\mathrm{C}_{10} \mathrm{H}_{10} \mathrm{O}_{4}$

Orthorhombic, $\mathrm{Pbca}$

$M_{r}=194.18$

Hall symbol: -P 2ac 2ab 
$a=15.269$ (4) $\AA$

$b=7.167(2) \AA$

$c=17.136(5) \AA$

$V=1875.2(9) \AA^{3}$

$Z=8$

$F(000)=816$

$D_{\mathrm{x}}=1.376 \mathrm{Mg} \mathrm{m}^{-3}$

Melting point: $381 \mathrm{~K}$

Data collection

Enraf-Nonius CAD-4

diffractometer

Radiation source: fine-focus sealed tube

Graphite monochromator

$\omega / 2 \theta$ scans

1718 measured reflections

1718 independent reflections

964 reflections with $I>2 \sigma(I)$

\section{Refinement}

Refinement on $F^{2}$

Least-squares matrix: full

$R\left[F^{2}>2 \sigma\left(F^{2}\right)\right]=0.047$

$w R\left(F^{2}\right)=0.119$

$S=1.02$

1718 reflections

167 parameters

0 restraints

Primary atom site location: structure-invariant direct methods
Mo $K \alpha$ radiation, $\lambda=0.71073 \AA$

Cell parameters from 25 reflections

$\theta=6.2-19.0^{\circ}$

$\mu=0.11 \mathrm{~mm}^{-1}$

$T=293 \mathrm{~K}$

Block, colourless

$0.42 \times 0.21 \times 0.15 \mathrm{~mm}$

$R_{\text {int }}=0.000$

$\theta_{\max }=25.3^{\circ}, \theta_{\min }=2.4^{\circ}$

$h=-18 \rightarrow 0$

$k=-8 \rightarrow 0$

$l=0 \rightarrow 20$

3 standard reflections every $60 \mathrm{~min}$

intensity decay: $1 \%$

Secondary atom site location: difference Fourier map

Hydrogen site location: inferred from neighbouring sites

All $\mathrm{H}$-atom parameters refined

$w=1 /\left[\sigma^{2}\left(F_{\mathrm{o}}^{2}\right)+(0.0516 P)^{2}\right]$

where $P=\left(F_{\mathrm{o}}^{2}+2 F_{\mathrm{c}}^{2}\right) / 3$

$(\Delta / \sigma)_{\max }<0.001$

$\Delta \rho_{\max }=0.17 \mathrm{e} \AA^{-3}$

$\Delta \rho_{\min }=-0.21$ e $\AA^{-3}$

Special details

Geometry. All e.s.d.'s (except the e.s.d. in the dihedral angle between two 1.s. planes) are estimated using the full covariance matrix. The cell e.s.d.'s are taken into account individually in the estimation of e.s.d.'s in distances, angles and torsion angles; correlations between e.s.d.'s in cell parameters are only used when they are defined by crystal symmetry. An approximate (isotropic) treatment of cell e.s.d.'s is used for estimating e.s.d.'s involving 1.s. planes.

Refinement. Refinement of $F^{2}$ against ALL reflections. The weighted $R$-factor $w R$ and goodness of fit $S$ are based on $F^{2}$, conventional $R$-factors $R$ are based on $F$, with $F$ set to zero for negative $F^{2}$. The threshold expression of $F^{2}>\sigma\left(F^{2}\right)$ is used only for calculating $R$-factors(gt) $e t c$. and is not relevant to the choice of reflections for refinement. $R$-factors based on $F^{2}$ are statistically about twice as large as those based on $F$, and $R$ - factors based on ALL data will be even larger.

Fractional atomic coordinates and isotropic or equivalent isotropic displacement parameters $\left(\hat{A}^{2}\right)$

\begin{tabular}{lllll}
\hline & $x$ & $y$ & $z$ & $U_{\text {iso }} * / U_{\text {eq }}$ \\
\hline H21a & $0.0220(14)$ & $0.495(4)$ & $0.3039(14)$ & $0.049(7)^{*}$ \\
H22b & $-0.1019(16)$ & $0.478(4)$ & $0.2296(16)$ & $0.063(9)^{*}$ \\
H22a & $-0.1276(16)$ & $0.540(4)$ & $0.3140(14)$ & $0.058(9)^{*}$ \\
H21b & $0.0046(17)$ & $0.281(4)$ & $0.2824(15)$ & $0.058(8)^{*}$ \\
H5 & $0.2323(19)$ & $0.109(4)$ & $0.5447(16)$ & $0.073(10)^{*}$ \\
H3 & $0.1355(15)$ & $0.315(4)$ & $0.3435(14)$ & $0.049(8)^{*}$ \\
H4 & $-0.1930(19)$ & $0.040(6)$ & $0.2804(19)$ & $0.104(13)^{*}$ \\
H4 & $0.2503(19)$ & $0.196(4)$ & $0.4169(15)$ & $0.058(9)^{*}$ \\
H6 & $0.092(2)$ & $0.133(4)$ & $0.6011(18)$ & $0.081(11)^{*}$
\end{tabular}




$\begin{array}{lllll}\text { H1 } & -0.100(2) & 0.276(4) & 0.5065(19) & 0.099(12)^{*} \\ \text { O3 } & -0.14623(11) & 0.1255(3) & 0.28392(12) & 0.0509(6) \\ \text { O2 } & -0.02664(10) & 0.3422(3) & 0.39246(10) & 0.0464(5) \\ \text { O4 } & -0.25446(11) & 0.3279(2) & 0.28021(11) & 0.0562(6) \\ \text { C23 } & -0.17679(16) & 0.2958(3) & 0.28326(14) & 0.0384(6) \\ \text { C2 } & 0.04663(17) & 0.2827(3) & 0.43064(16) & 0.0414(7) \\ \text { C1 } & 0.03508(17) & 0.2307(4) & 0.50805(16) & 0.0443(7) \\ \text { C21 } & -0.01910(17) & 0.3884(4) & 0.31172(16) & 0.0426(7) \\ \text { O1 } & -0.06445(15) & 0.2082(3) & 0.61361(12) & 0.0779(7) \\ \text { C22 } & -0.10849(17) & 0.4449(4) & 0.28378(19) & 0.0421(7) \\ \text { C3 } & 0.12868(17) & 0.2735(4) & 0.39650(18) & 0.0511(8) \\ \text { C6 } & 0.1071(2) & 0.1672(5) & 0.5502(2) & 0.0625(9) \\ \text { C11 } & -0.0514(2) & 0.2416(4) & 0.54553(18) & 0.0565(8) \\ \text { C4 } & 0.1983(2) & 0.2109(5) & 0.4398(2) & 0.0690(10) \\ \text { C5 } & 0.1876(2) & 0.1563(6) & 0.5161(2) & 0.0761(11)\end{array}$

Atomic displacement parameters $\left(\AA^{2}\right)$

\begin{tabular}{lllllll}
\hline & $U^{11}$ & $U^{22}$ & $U^{33}$ & $U^{12}$ & $U^{13}$ & $U^{23}$ \\
\hline O3 & $0.0400(10)$ & $0.0306(11)$ & $0.0823(15)$ & $0.0022(8)$ & $0.0009(11)$ & $0.0024(10)$ \\
O2 & $0.0428(10)$ & $0.0563(12)$ & $0.0401(11)$ & $0.0032(9)$ & $0.0023(9)$ & $0.0062(10)$ \\
O4 & $0.0409(10)$ & $0.0359(10)$ & $0.0919(15)$ & $0.0035(9)$ & $-0.0046(11)$ & $-0.0016(11)$ \\
C23 & $0.0428(15)$ & $0.0324(14)$ & $0.0401(15)$ & $0.0047(12)$ & $0.0018(13)$ & $0.0013(12)$ \\
C2 & $0.0481(16)$ & $0.0314(14)$ & $0.0449(17)$ & $-0.0040(12)$ & $-0.0047(13)$ & $-0.0030(12)$ \\
C1 & $0.0533(18)$ & $0.0353(15)$ & $0.0442(17)$ & $-0.0071(13)$ & $-0.0018(13)$ & $-0.0035(13)$ \\
C21 & $0.0438(16)$ & $0.0416(17)$ & $0.0424(16)$ & $-0.0065(14)$ & $0.0018(13)$ & $0.0020(14)$ \\
O1 & $0.1120(18)$ & $0.0752(16)$ & $0.0466(14)$ & $-0.0116(14)$ & $0.0176(12)$ & $0.0007(12)$ \\
C22 & $0.0500(16)$ & $0.0312(16)$ & $0.0451(18)$ & $-0.0034(12)$ & $-0.0004(15)$ & $0.0009(14)$ \\
C3 & $0.0437(16)$ & $0.0558(18)$ & $0.0537(19)$ & $-0.0044(14)$ & $-0.0007(16)$ & $0.0009(16)$ \\
C6 & $0.075(2)$ & $0.059(2)$ & $0.054(2)$ & $-0.0036(18)$ & $-0.011(2)$ & $-0.0018(17)$ \\
C11 & $0.077(2)$ & $0.0448(19)$ & $0.047(2)$ & $-0.0066(17)$ & $0.0067(18)$ & $-0.0035(15)$ \\
C4 & $0.0453(19)$ & $0.084(3)$ & $0.077(3)$ & $0.0022(18)$ & $-0.0088(19)$ & $-0.009(2)$ \\
C5 & $0.072(3)$ & $0.080(3)$ & $0.077(3)$ & $0.009(2)$ & $-0.031(2)$ & $0.000(2)$ \\
& & & & & &
\end{tabular}

Geometric parameters $\left(\AA,{ }^{\circ}\right)$

\begin{tabular}{llll}
\hline $\mathrm{O} 3-\mathrm{C} 23$ & $1.306(3)$ & $\mathrm{C} 21-\mathrm{H} 21 \mathrm{~b}$ & $0.99(3)$ \\
$\mathrm{O} 3-\mathrm{H} 41$ & $0.95(4)$ & $\mathrm{O} 1-\mathrm{C} 11$ & $1.208(3)$ \\
$\mathrm{O} 2-\mathrm{C} 2$ & $1.364(3)$ & $\mathrm{C} 22-\mathrm{H} 22 \mathrm{~b}$ & $0.96(3)$ \\
$\mathrm{O} 2-\mathrm{C} 21$ & $1.427(3)$ & $\mathrm{C} 22-\mathrm{H} 22 \mathrm{a}$ & $0.90(3)$ \\
$\mathrm{O} 4-\mathrm{C} 23$ & $1.209(3)$ & $\mathrm{C} 3-\mathrm{C} 4$ & $1.371(4)$ \\
$\mathrm{C} 23-\mathrm{C} 22$ & $1.493(3)$ & $\mathrm{C} 3-\mathrm{H} 3$ & $0.96(2)$ \\
$\mathrm{C} 2-\mathrm{C} 3$ & $1.384(4)$ & $\mathrm{C} 6-\mathrm{C} 5$ & $1.363(5)$ \\
$\mathrm{C} 2-\mathrm{C} 1$ & $1.389(4)$ & $\mathrm{C} 6-\mathrm{H} 6$ & $0.93(3)$ \\
$\mathrm{C} 1-\mathrm{C} 6$ & $1.392(4)$ & $\mathrm{C} 11-\mathrm{H} 1$ & $1.03(3)$ \\
$\mathrm{C} 1-\mathrm{C} 11$ & $1.471(4)$ & $\mathrm{C} 4-\mathrm{C} 5$ & $1.375(5)$ \\
$\mathrm{C} 21-\mathrm{C} 22$ & $1.502(4)$ & $\mathrm{C} 4-\mathrm{H} 4$ & $0.89(3)$ \\
$\mathrm{C} 21-\mathrm{H} 21 \mathrm{a}$ & $1.00(3)$ & $\mathrm{C} 5-\mathrm{H} 5$ & $0.91(3)$
\end{tabular}




\begin{tabular}{|c|c|c|c|}
\hline $\mathrm{C} 23-\mathrm{O} 3-\mathrm{H} 41$ & $110(2)$ & $\mathrm{C} 21-\mathrm{C} 22-\mathrm{H} 22 \mathrm{~b}$ & $106.2(15)$ \\
\hline $\mathrm{C} 2-\mathrm{O} 2-\mathrm{C} 21$ & $118.1(2)$ & $\mathrm{C} 23-\mathrm{C} 22-\mathrm{H} 22 \mathrm{a}$ & $108.5(17)$ \\
\hline $\mathrm{O} 4-\mathrm{C} 23-\mathrm{O} 3$ & $121.9(2)$ & $\mathrm{C} 21-\mathrm{C} 22-\mathrm{H} 22 \mathrm{a}$ & $108.3(17)$ \\
\hline $\mathrm{O} 4-\mathrm{C} 23-\mathrm{C} 22$ & $123.3(2)$ & $\mathrm{H} 22 \mathrm{~b}-\mathrm{C} 22-\mathrm{H} 22 \mathrm{a}$ & $113(2)$ \\
\hline $\mathrm{O} 3-\mathrm{C} 23-\mathrm{C} 22$ & $114.8(2)$ & $\mathrm{C} 4-\mathrm{C} 3-\mathrm{C} 2$ & $119.2(3)$ \\
\hline $\mathrm{O} 2-\mathrm{C} 2-\mathrm{C} 3$ & $123.7(3)$ & $\mathrm{C} 4-\mathrm{C} 3-\mathrm{H} 3$ & $121.8(14)$ \\
\hline $\mathrm{O} 2-\mathrm{C} 2-\mathrm{C} 1$ & $116.0(2)$ & $\mathrm{C} 2-\mathrm{C} 3-\mathrm{H} 3$ & $118.9(15)$ \\
\hline $\mathrm{C} 3-\mathrm{C} 2-\mathrm{C} 1$ & $120.4(3)$ & $\mathrm{C} 5-\mathrm{C} 6-\mathrm{C} 1$ & $120.6(4)$ \\
\hline $\mathrm{C} 6-\mathrm{C} 1-\mathrm{C} 2$ & $118.9(3)$ & $\mathrm{C} 5-\mathrm{C} 6-\mathrm{H} 6$ & $127(2)$ \\
\hline $\mathrm{C} 6-\mathrm{C} 1-\mathrm{C} 11$ & $120.0(3)$ & $\mathrm{C} 1-\mathrm{C} 6-\mathrm{H} 6$ & $112.3(19)$ \\
\hline $\mathrm{C} 2-\mathrm{C} 1-\mathrm{C} 11$ & $121.1(3)$ & $\mathrm{O} 1-\mathrm{C} 11-\mathrm{C} 1$ & $124.0(3)$ \\
\hline $\mathrm{O} 2-\mathrm{C} 21-\mathrm{C} 22$ & $107.3(2)$ & $\mathrm{O} 1-\mathrm{C} 11-\mathrm{H} 1$ & $123.9(19)$ \\
\hline $\mathrm{O} 2-\mathrm{C} 21-\mathrm{H} 21 \mathrm{a}$ & $111.0(14)$ & $\mathrm{C} 1-\mathrm{C} 11-\mathrm{H} 1$ & $112.0(19)$ \\
\hline $\mathrm{C} 22-\mathrm{C} 21-\mathrm{H} 21 \mathrm{a}$ & $108.9(14)$ & $\mathrm{C} 3-\mathrm{C} 4-\mathrm{C} 5$ & $121.0(3)$ \\
\hline $\mathrm{O} 2-\mathrm{C} 21-\mathrm{H} 21 \mathrm{~b}$ & $110.0(16)$ & $\mathrm{C} 3-\mathrm{C} 4-\mathrm{H} 4$ & $119.5(18)$ \\
\hline $\mathrm{C} 22-\mathrm{C} 21-\mathrm{H} 21 \mathrm{~b}$ & $112.4(15)$ & $\mathrm{C} 5-\mathrm{C} 4-\mathrm{H} 4$ & $119.2(18)$ \\
\hline $\mathrm{H} 21 \mathrm{a}-\mathrm{C} 21-\mathrm{H} 21 \mathrm{~b}$ & $107(2)$ & $\mathrm{C} 6-\mathrm{C} 5-\mathrm{C} 4$ & $119.9(4)$ \\
\hline $\mathrm{C} 23-\mathrm{C} 22-\mathrm{C} 21$ & $116.3(2)$ & $\mathrm{C} 6-\mathrm{C} 5-\mathrm{H} 5$ & $118.0(19)$ \\
\hline $\mathrm{C} 23-\mathrm{C} 22-\mathrm{H} 22 \mathrm{~b}$ & $104.2(16)$ & $\mathrm{C} 4-\mathrm{C} 5-\mathrm{H} 5$ & $122.1(19)$ \\
\hline $\mathrm{C} 21-\mathrm{O} 2-\mathrm{C} 2-\mathrm{C} 3$ & $3.4(4)$ & $\mathrm{O} 2-\mathrm{C} 2-\mathrm{C} 3-\mathrm{C} 4$ & $-179.7(3)$ \\
\hline $\mathrm{C} 21-\mathrm{O} 2-\mathrm{C} 2-\mathrm{C} 1$ & $-176.9(2)$ & $\mathrm{C} 1-\mathrm{C} 2-\mathrm{C} 3-\mathrm{C} 4$ & $0.6(4)$ \\
\hline $\mathrm{O} 2-\mathrm{C} 2-\mathrm{C} 1-\mathrm{C} 6$ & $179.5(2)$ & $\mathrm{C} 2-\mathrm{C} 1-\mathrm{C} 6-\mathrm{C} 5$ & $0.0(4)$ \\
\hline $\mathrm{C} 3-\mathrm{C} 2-\mathrm{C} 1-\mathrm{C} 6$ & $-0.8(4)$ & $\mathrm{C} 11-\mathrm{C} 1-\mathrm{C} 6-\mathrm{C} 5$ & $179.9(3)$ \\
\hline $\mathrm{O} 2-\mathrm{C} 2-\mathrm{C} 1-\mathrm{C} 11$ & $-0.4(4)$ & $\mathrm{C} 6-\mathrm{C} 1-\mathrm{C} 11-\mathrm{O} 1$ & $4.3(4)$ \\
\hline $\mathrm{C} 3-\mathrm{C} 2-\mathrm{C} 1-\mathrm{C} 11$ & $179.3(3)$ & $\mathrm{C} 2-\mathrm{C} 1-\mathrm{C} 11-\mathrm{O} 1$ & $-175.8(3)$ \\
\hline $\mathrm{C} 2-\mathrm{O} 2-\mathrm{C} 21-\mathrm{C} 22$ & $178.8(2)$ & $\mathrm{C} 2-\mathrm{C} 3-\mathrm{C} 4-\mathrm{C} 5$ & $0.4(5)$ \\
\hline $\mathrm{O} 4-\mathrm{C} 23-\mathrm{C} 22-\mathrm{C} 21$ & $162.1(3)$ & $\mathrm{C} 1-\mathrm{C} 6-\mathrm{C} 5-\mathrm{C} 4$ & $1.0(5)$ \\
\hline $\mathrm{O} 3-\mathrm{C} 23-\mathrm{C} 22-\mathrm{C} 21$ & $-20.0(4)$ & $\mathrm{C} 3-\mathrm{C} 4-\mathrm{C} 5-\mathrm{C} 6$ & $-1.2(6)$ \\
\hline $\mathrm{O} 2-\mathrm{C} 21-\mathrm{C} 22-\mathrm{C} 23$ & $-65.9(3)$ & & \\
\hline
\end{tabular}

Hydrogen-bond geometry $\left(\AA,{ }^{\circ}\right)$

\begin{tabular}{lllll}
\hline$D-\mathrm{H} \cdots A$ & $D-\mathrm{H}$ & $\mathrm{H} \cdots A$ & $D \cdots A$ & $D-\mathrm{H} \cdots A$ \\
\hline $\mathrm{C} 6-\mathrm{H} 6 \cdots \mathrm{O} 1$ & $0.94(3)$ & $2.46(3)$ & $2.851(4)$ & $105(2)$ \\
$\mathrm{C} 11-\mathrm{H} 1 \cdots \mathrm{O} 2$ & $1.03(3)$ & $2.30(3)$ & $2.746(4)$ & $105(2)$ \\
$\mathrm{O} 3-\mathrm{H} 41 \cdots \mathrm{O} 4^{\mathrm{i}}$ & $0.94(4)$ & $1.72(4)$ & $2.618(3)$ & $158(3)$ \\
$\mathrm{C} 21-\mathrm{H} 21 a \cdots{ }^{\mathrm{ii}}$ & $1.00(3)$ & $2.64(3)$ & $3.409(4)$ & $134.5(2)$ \\
$\mathrm{C} 22-\mathrm{H} 22 b \cdots{ }^{\mathrm{iii}}$ & $0.96(3)$ & $2.46(3)$ & $3.187(4)$ & $132(2)$ \\
$\mathrm{C} 21-\mathrm{H} 21 a \cdots{ }^{i i}$ & $1.00(3)$ & $2.60(2)$ & $3.456(3)$ & $144(2)$ \\
$\mathrm{C} 3-\mathrm{H} 3 \cdots{ }^{\text {iv }}$ & $0.96(2)$ & $2.71(2)$ & $3.536(4)$ & $144.9(2)$ \\
\hline
\end{tabular}

Symmetry codes: (i) $-x-1 / 2, y-1 / 2, z$; (ii) $-x,-y+1,-z+1$; (iii) $x,-y+1 / 2, z-1 / 2$; (iv) $-x, y+1 / 2,-z+1 / 2$; (v) $x+1 / 2, y,-z+1 / 2$. 